\title{
Costos de hospitalización de los accidentes por mordedura de canes en un hospital de niños en Lima- Perú, periodo 2006-2010
}

\author{
Cost of hospitalization for dogs bite accidents in a children's hospital in Lima, Peru, 2006-2010 period
}

\author{
Claudia Romero ${ }^{1}$, Henry Hernández ${ }^{2}$, Néstor Falcón ${ }^{1}$
}

\section{RESUMEN}

Objetivos: Determinar los costos de los pacientes hospitalizados por mordedura de canes atendidos en el Instituto Nacional de Salud del Niño Lima- Perú (INSN), periodo 2006-2010. Material y Métodos: Se revisó las historias clínicas de 72 pacientes con diagnóstico de mordedura canina de las cuales se obtuvo información tal como sexo, procedencia, edad, estancia hospitalaria, medicamentos usados, exámenes auxiliares y procedimientos médicos. Los costos fueron proporcionados por el Servicio de Farmacia y el Servicio de Cirugía Plástica del INSN. Resultados: Del total de hospitalizados, $68 \%$ pertenecieron al sexo masculino y $32 \%$ al femenino. El $79 \%$ de pacientes asumieron los costos hospitalarios mediante financiamiento propio, mientras, que en el $21 \%$ de los pacientes el costo fue subvencionado por el INSN. El 78\% de los casos pertenecieron a Lima y Callao y el 22\% a regiones del interior del país. El rango de edad de los pacientes afectados fue de 1 a 4 años (53\%) y el promedio de estancia hospitalaria fue de 7 días, con un rango de 1 a 32 días. Se obtuvo que el costo total fue de 15 181,5 US dólares y el costo promedio por día fue de 30,6 US dólares. El rubro en el que se produjo el mayor costo es el de farmacia en el que se encuentra incluido principalmente materiales quirúrgicos y productos de fluidoterapia. Conclusiones: Los accidentes por mordedura canina producen costos de hospitalización elevados, por lo que se debe establecer programas de control a fin de disminuir su incidencia.

PALABRAS CLAVE: Accidentes, mordedura canina, costos de hospitalización, pacientes.

\section{SUMMARY}

Objectives: Dog bites are an important problem in public health which involves physical and physiological injuries, zoonotics diseases and mean a high cost in the community. For this reason the objective of this research was to define the costs of hospitalization for patients at Instituto Nacional de Salud del Niño Lima-Perú (INSN), during the 2006-2010 period. Material and methods: Medical history of 72 patients diagnosed with dog bites were reviewed in which we obtained information about sex, place of origin, age, hospital staying, drugs used, tests, and surgical procedures. Cost information was provided by the Pharmacy Service and Plastic Surgery Service of INSN. Results: From all the hospitalized, $68 \%$ were male and $32 \%$ were female. $79 \%$ of patients assumed the cost of hospitalization through their own funding and for $21 \%$ of the patients the cost was subsidized by the INSN. $78 \%$ of the cases belonged to Lima and Callao and $22 \%$ to the country's interior regions. The age range of affected patients was from 1 to 4 years (53\%). The average hospital staying was 7 days, with a range from 1 to 32 days. The total cost was 15 181,5. US dollars and the average cost per day was 30,6 US dollars. The item which produces the major cost is the pharmacy (involving surgical and fluid products). Conclusions: Dog bites accidents produce high hospital cost, so

Facultad de Medicina Veterinaria y Zootecnia, Universidad Peruana Cayetano Heredia. Lima, Perú.

Estrategia Sanitaria de Zoonosis - Oficina de Epidemiologia - Instituto Nacional de Salud del Niño. Lima, Perú. 
it must be established control programs to reduce its incidence.

KEY WORDS: accidents, dog bites, costs of hospitalization, patients.

\section{INTRODUCCIÓN}

La convivencia entre el hombre y canes, habitualmente beneficiosa para ambos, tiene su lado negativo en la zoonosis y las lesiones producidas, especialmente las mordeduras (1). Estas representan una de las causas más frecuentes de morbilidad e incapacidad y constituyen un problema global de salud pública (2-5).

Se ha demostrado que muchas de las interacciones entre canes y niños que terminan en accidentes son provocadas por los niños. Las regiones anatómicas más afectadas suelen ser la cabeza y cara (6); pudiendo convertirse en víctimas fatales ya sea por la presión ejercida por la quijada de un perro adulto o por la hemorragia producida al dañar un vaso de la cabeza o del cuello (7).

Las mordeduras caninas pueden ser de tipo sorpresivo o provocado. Un estudio retrospectivo realizado en el Instituto Nacional de Salud del Niño durante el periodo 1995-2009, encontró que los accidentes más frecuentes se producían en el domicilio del perro y eran de tipo provocado $(66,5 \%)$ (6). Los accidentes de tipo sorpresivos suelen ser más frecuentes en la vía pública y se producen cuando los canes se sienten amenazados o tienen miedo (8).

Las principales consecuencias que se derivan de las mordeduras de canes son las lesiones y cicatrices producidas por las agresiones, transmisión de enfermedades infecciosas (rabia, pasteurelosis, tétanos y otras infecciones secundarias), secuelas psicológicas, incapacidades, costes económicos derivados de los tratamientos médicos y psicológicos, pérdida de clases en niños o jornada laborales en adultos, el control de animales y la muerte de la víctima causada por las lesiones producidas a causa de la agresión o por complicaciones o enfermedades transmitidas por la mordedura $(5,9-10)$.

Los accidentes por mordedura de canes en Estados Unidos ocasionan un costo promedio de hospitalización calculado en 18200 US dólares, 50\% más alto que el costo de hospitalización por lesiones diferentes (12 100 US dólares) (11). Asimismo, la industria de seguros estima que gasta más de 1 billón de dólares cubriendo los accidentes por mordedura de canes (12).

Los accidentes por mordedura de canes han aumentado sobre todo en los países donde el control de la población canina es tan deficiente como el nuestro. La prevención de los mismos requiere de la educación dentro del manejo de poblaciones caninas, ya que el comportamiento humano es un factor influyente en la dinámica de las poblaciones de canes. A fin de evaluar el impacto económico de este problema en nuestro país, el objetivo del estudio fue determinar los costos directos promedio de los casos de mordeduras de canes que requirieron hospitalización durante el periodo 2006-2010 en el Instituto Nacional de Salud del Niño Lima- Perú (INSN).

\section{MATERIALES Y MÉTODOS}

La recolección de datos de los casos hospitalizados por mordedura canina en el periodo 2006 - 2010, se llevó a cabo a través de la revisión del archivo de historias clínicas en la Estrategia Sanitaria de Zoonosis de la Oficina de Epidemiología del Instituto Nacional de Salud del Niño (INSN), ubicado en la Av. Brasil 642-Breña, Lima. El diseño de la base de datos y el análisis estadístico se realizó en la Facultad de Medicina Veterinaria y Zootecnia de la Universidad Peruana Cayetano Heredia.

El tipo de investigación correspondió a un estudio descriptivo, observacionaly retrospectivo. Lapoblación objetivo estuvo conformada por todos los accidentes de mordedura canina que fueron hospitalizados entre los años 2006 - 2010. Se excluyeron aquellas historias clínicas que no se encontraron legibles y/o que no contengan información disponible, en formato físico o electrónico, así como también aquellas que fueron depuradas por pertenecer a pacientes que en el año del estudio (2011) habían superado la edad de atención en el INSN (17 años, 11 meses, 29 días).

Las variables en estudio fueron las siguientes:

- Datos generales del paciente agredido (género, edad, procedencia, tiempo de estancia hospitalaria). - Variables relacionadas a la lesión (región anatómica afectada). 
Variables relacionadas a la Fuente de Financiamiento:

- Caso subvencionado: costo hospitalario asumido por el INSN.

Caso pagante: pacientes que asumieron el costo de la atención hospitalaria.

Variables relacionadas a los costos de hospitalización: costo de estancia hospitalaria, costo de exámenes auxiliares, costo de farmacia, costo por tipo de atención quirúrgica.

La información demográfica, de estancia hospitalaria, tipo de exámenes auxiliares, atención quirúrgica y farmacia, se obtuvo a través de la Unidad de Registros Médicos de la Oficina de Estadística e Informática del INSN. Los costos de los servicios y farmacia requeridos fueron proporcionados por el Servicio de Farmacia y Servicio de Cirugía Plástica del INSN (Tabla 1).

Se caracterizó demográficamente a la población en estudio y se determinó el costo promedio, desviación estándar, valores máximos y mínimos, por día de hospitalización, por región anatómica afectada, costos de estancia hospitalaria, exámenes auxiliares, farmacia, atención quirúrgica y el costo total de casos por años y por periodo (acumulado). Además, se registraron los fármacos, exámenes auxiliares y tipo de atención quirúrgica más usados en el tratamiento de las mordeduras caninas.

Todos los procedimientos del presente estudio trataron de preservar la integridad y los derechos fundamentales de los pacientes sujetos a investigación. El estudio siguió las normas éticas del Comité de Ética de la Universidad Peruana Cayetano Heredia.

\section{RESULTADOS}

El total de pacientes incluidos en el estudio fueron 72. El 68\% (49 casos) pertenecieron al sexo masculino y $32 \%$ ( 23 casos) al sexo femenino. Con respecto a la procedencia $78 \%$ (56 pacientes) pertenecieron a Lima y Callao y 22\% (16 pacientes) a regiones del interior del país. El rango de edad de los pacientes más afectados fue de 1 a 4 años (53\%), de 5 a 9 años $(32 \%)$ y de 10 a 17 años (15\%). El promedio de estancia hospitalaria fue de 7 días, con un rango de 1 a 32 días. El costo total de hospitalización de las atenciones de los accidentes por mordedura de canes para esta población fue de 15182 US dólares y el costo promedio por día de hospitalización fue de 31 US dólares.

El mayor costo de hospitalización correspondió a farmacia, el cual incluyó el $46,8 \%$ del costo total, dentro del cual el costo de materiales para cirugía y productos de fluidoterapia fueron los más altos. Después siguen los costos por atención quirúrgica, estancia hospitalaria y exámenes auxiliares que correspondieron al 29,6\%, 13,6 \% y 10,1\% del costo total respectivamente (Tabla 2).

Dentro de los costos de farmacia se consideró el costo de antibióticos, antiinflamatorios, analgésicos y otros. En los costos de farmacia también se encuentran los materiales para cirugía. Los más usados fueron los guantes quirúrgicos, sonda de aspiración, jeringas descartables y hojas de bisturí; por otro lado, en productos para fluidoterapia los más usados fueron dextrosa al 5\%, $\mathrm{Cl} \mathrm{Na} 20 \%$ y $\mathrm{Cl} \mathrm{Na}$ al $9 \%$. Los exámenes auxiliares más usados en los casos de mordedura canina fueron hemograma completo, grupo sanguíneo y coagulación - sangría. Con respecto al tipo de atención quirúrgica el procedimiento de

Tabla 1. Distribución de costos (US dólares) de hospitalización de pacientes con mordedura de canes. Instituto Nacional de Salud del Niño Lima- Perú, Periodo 2006-2010

\begin{tabular}{|c|c|c|c|c|c|c|c|}
\hline \multirow{2}{*}{$\begin{array}{l}\text { Tipo de } \\
\text { Costo }\end{array}$} & \multirow{2}{*}{ Media } & \multirow{2}{*}{ Mediana } & \multirow{2}{*}{ Moda } & \multirow{2}{*}{$\begin{array}{c}\text { Desviación } \\
\text { Estándar }\end{array}$} & \multicolumn{2}{|c|}{ Valores extremos } & \multirow{2}{*}{$\begin{array}{l}\text { Costo } \\
\text { Total }\end{array}$} \\
\hline & & & & & Mínimo & Máximo & \\
\hline Estancia hospitalaria & 28,6 & 16,6 & 4,2 & 85,1 & 4,2 & 132,8 & 2058,9 \\
\hline Farmacia & 98,6 & 64,4 & --- & 115,2 & 7,0 & 787,5 & 7097,5 \\
\hline Exámenes auxiliares & 22,3 & 17,7 & 6,0 & 14,9 & 5,7 & 77,4 & 1535,6 \\
\hline Atención quirúrgica & 71,6 & 69,2 & 43,4 & 34,3 & 28,3 & 157,7 & 4489,6 \\
\hline
\end{tabular}


Tabla 2. Estancia hospitalaria y distribución de costos (US dólares) por día según ubicación de la lesión causada por los accidentes por mordedura de canes. Instituto Nacional de Salud del Niño Lima- Perú, Periodo 2006-2010.

\begin{tabular}{lccc}
\hline \multirow{2}{*}{ Variable } & \multicolumn{3}{c}{$\begin{array}{c}\text { Ubicación Anatómica de la Lesión } \\
\text { (Número de Casos) }\end{array}$} \\
\cline { 2 - 4 } & CABEZA & MIEMBRO & OTROS \\
\cline { 2 - 4 } & $\mathbf{( 6 1 )}$ & 9 & 14 \\
Media estancia hospitalaria (días) & 6 & 4,2 & 4,2 \\
Costo de estancia hospitalaria & 4,2 & 17,8 & 8,7 \\
Costo de farmacia & 14,6 & 2,5 & 1,8 \\
Costo de exámenes auxiliares & 3,4 & 8,0 & 5,2 \\
Costo de Atención Quirúrgica & 9,8 & 32,5 & 19,9 \\
\hline
\end{tabular}

Tabla 3. Fuente de financiamiento y ubicación de la lesión de los accidentes por mordedura de canes. Instituto Nacional de Salud del Niño Lima- Perú, Periodo 2006-2010.

\begin{tabular}{lcc}
\hline Ubicación de Lesión & Número de Casos & Costo Total \\
\hline SUBVENCIONADO: & 12 & 3591,8 \\
Cabeza & 1 & 44,2 \\
Miembros & 2 & 472,0 \\
Otros & & \\
PAGANTE: & 49 & 8400,5 \\
Cabeza & 6 & 2008,6 \\
Miembros & 2 & 664,5 \\
Otros & & \\
\hline
\end{tabular}

*Otros: genitales/región anal, tórax y abdomen

curación y sutura fue el más aplicado. De los 72 pacientes, 63 requirieron de cirugía $(87,5 \%)$ y sólo 9 $(12,5 \%)$ no necesitaron de ninguna operación.

Respecto al costo total por lugar de procedencia de los pacientes con diagnóstico de mordedura canina, se hospitalizaron 56 pacientes procedentes de Lima y Callao en el INSN, lo cual representó un costo total de 11122 US dólares. Los casos hospitalizados procedentes de regiones del interior del país fueron 16 , siendo el costo total de 4059 US dólares.

El costo total de hospitalización subvencionado de 15 pacientes durante el periodo de estudio fue de 4108 US dólares ( $27 \%$ del costo total). La diferencia (57 pacientes) fue asumida por el paciente y corresponde a un total de 11074 US dólares ( $73 \%$ del costo total) (Tabla 3).

\section{DISCUSIÓN}

Los costos económicos de la atención y tratamiento a los pacientes con mordedura canina representan una carga para los servicios de salud, los pacientes y sus familias. A través del estudio se pudo conocer el costo de hospitalización, es decir los costos directos totales de la atención de los pacientes con mordedura canina en el INSN, información que hasta la actualidad no se había evaluado. Una valorización de todos los casos que se producen en un año siguiendo el modelo de este estudio, puede servir para ser comparado con los costos de estrategias de prevención de las mordeduras caninas, y de esta manera se podría contrastar la factibilidad de invertir en campañas de prevención y control, en vez de seguir atendiendo más casos.

El costo directo promedio/día de hospitalización 
por ubicación de la lesión por mordeduras en miembros son sólo 0,75 US dólares más que en cabeza a pesar de haberse presentado en pocos casos. Para el caso de miembros, el costo promedio/día de farmacia eleva el costo promedio/día total, esto puede deberse a que la recuperación de las mismas es más lenta (13), por lo tanto hay más días de hospitalización y más uso de medicinas y materiales de cirugía. Este costo por día representa para las familias de bajos estratos económicos una carga alta la cual no es de fácil disposición.

El costo promedio/día de la atención hospitalaria distribuido según sexo no reveló diferencias. En el caso de los grupos etáreos, existe una tendencia a que el costo promedio/día sea mayor entre los afectados de 10 a 17 años. Esto se debería a que en más de la mitad de los casos el tiempo de estancia hospitalaria fue superior al tiempo promedio, lo cual está relacionado a que en la mayoría de los casos los pacientes sufrieron lesiones múltiples en cabeza y miembros, por lo tanto los días de recuperación y el tratamiento incrementaron el costo total.

De acuerdo al artículo 14 de la Ley $\mathrm{N}^{\circ} 27596$ (14), si un can ocasiona una lesión grave a una persona, el dueño del can está obligado a asumir el costo total de la hospitalización, medicamentos y cirugía necesaria hasta la recuperación del agredido. Sin embargo, según la revisión de las historias clínicas sólo en un caso el dueño del animal agresor asumió el costo de farmacia, por lo tanto un porcentaje importante de pacientes o familiares $(79 \%)$ tienen que desviar recursos no previstos a gastos por hospitalización, farmacia, atenciones quirúrgicas, etc., privándose de otras necesidad y/o endeudándose.

Se entiende que los afectados recurren al INSN no solo por que se ofrece una atención especializada, sino porque al ser una entidad estatal, el costo de las atenciones es más bajo que en los servicios particulares de salud. Para los casos subvencionados (21\%) por el INSN, estos se sustentaron en una evaluación socioeconómica mediante la asistenta social, la cual incluyó a pacientes de extrema pobreza y a pacientes abandonados por sus apoderados.

El costo directo de hospitalización más elevado fue el de farmacia, que representa un $46.8 \%$ del costo total, siendo el costo de materiales de cirugía y fluidoterapia los más altos, esto debido a que la mayoría de los casos, al ser de gravedad, necesitaron estabilizar al paciente y después requirieron de un tratamiento quirúrgico.
La mayor cantidad de pacientes procedieron de Lima. Ello se explica por la accesibilidad al centro hospitalario. Los casos que proceden de regiones son aquellos casos graves que son transferidos al INSN para recibir una atención especializada (6). El costo total de los pacientes procedentes de regiones del interior del país representa el $27 \%$ del costo total. Este dato es significativo y debe servir de información a las autoridades del gobierno para mejorar las atenciones de cada caso en los lugares que ocurren los accidentes.

En este estudio el promedio de estancia hospitalaria fue de 7 días, con un rango de 1 a 32 días, en comparación con la estancia promedio de hospitalización por accidente de mordedura canina en USA que fue de 3,3 días (11). Esta diferencia se puede deber a que en este estudio la mayoría de casos sufrieron mordedura en cara y extremidades consideradas de tipo grave a nivel estético, lo cual hacia que el tratamiento sea largo y se necesiten de más días para la recuperación. Otra causa que aumenta el tiempo de hospitalización es que las suturas de las heridas causadas por la mordedura de un animal en primera intención se pueden contaminar. Pero, tratarla a base de curaciones y cerrarla de tercera intención requiere de mayores días de hospitalización ya que se necesitan de procedimientos más complicados (15). Sin embargo esta situación fue la menos frecuente en el estudio.

Los pacientes que son hospitalizados o remitidos a especialistas suelen ser los que presentan lesiones graves en las manos, infección extensa, lesión en el tendón, cartílago, hueso o cápsula articular, desfiguraciones o pérdida de tejidos que requieren cirugía y niños pequeños con heridas en la cabeza provocadas por canes de gran tamaño (16). En el estudio fueron 72 los pacientes hospitalizados por presentar lesiones graves en cabeza y extremidades, de las cuales 63 necesitaron ser operados mediante cirugía especializada.

Una de las consecuencias importantes que supone la mordedura de un animal es la tendencia a la infección de la herida. Aproximadamente el $20 \%$ de las mordeduras caninas tienen el riesgo de infectarse. Es por eso que se requiere del uso de antibióticos de manera profiláctica para prevenir el ingreso de organismos extraños al cuerpo como la flora normal anaerobia de la cavidad bucal del perro, en la cual se encuentra Pasteurella multocida, S. viridans, $S$. aureus, así como Bacteroides y Fusobacterias (17-18), además de la flora aerobia de la piel de la víctima que 
puede inducir infección (19-20).

Los antibióticos que se recomiendan tanto en profilaxis como en tratamiento son los siguientes: amoxicilina más ácido clavulánico y como alternativa, clindamicina asociada con levofloxacina, o monoterapias a base de moxifloxacina, imipenem o meropenem $(5,15)$. En el estudio los antibióticos más utilizados fueron amikacina, clindamicina, amoxicilina más ácido clavulánico, oxacilina y ceftriazona, siendo la amoxicilina asociada con ácido clavulánico la de mayor uso en los pacientes y la más recomendada $(7,21)$. Teniendo el conocimiento de los fármacos más utilizados en el tratamiento profiláctico de las mordeduras caninas, el INSN podrá abastecer estas medicinas para la atención inmediata del paciente, asegurando y acortando la recuperación del mismo.

El $87,5 \%$ (63 casos) necesitaron ser operados. Los procedimientos quirúrgicos más aplicados en estos casos de mordedura canina fueron limpieza quirúrgica y sutura, limpieza quirúrgica y plastía, y limpieza quirúrgica e injerto de piel. Ya que estos procedimientos de cirugía plástica deben ser tratados con seguridad, cada paciente debe pasar por un examen de riesgo quirúrgico. Es así que los exámenes auxiliares que se realizaron en la mayoría de los casos fueron: hemograma completo, grupo sanguíneo y coagulación y sangría.

Dada la gravedad de los casos hospitalizados, el gasto económico para familias de estratos económicos bajos y el incumplimiento de las leyes por parte de los dueños de canes agresores, se debe seguir trabajando en planes de prevención basado en la educación sobre la tenencia responsable de animales para la comunidad, con la finalidad de disminuir la casuística, además de la aplicación de las sanciones pertinentes para aquellas personas que infrinjan la legislación correspondiente a este tema.

\section{CONCLUSIONES}

El estudio de costos de hospitalización de los accidentes por mordedura canina en el INSN, durante el periodo 2006-2010 concluye que:

El costo promedio/día de hospitalización significa un importante gasto para las familias de bajos estratos económicos, las cuales deben de asumir esos gastos no previstos para la recuperación del paciente.

El rubro en el que se produce el mayor costo es el de farmacia, en el que se encuentra incluido principalmente materiales quirúrgicos y productos de fluidoterapia.

\section{Correspondencia}

Claudia Romero Aliaga

Correo electrónico: claudia.romero@upch.pe

\section{REFERENCIAS BIBLIOGRAFICAS}

1. Schvartzman S, Pacín M. Lesiones por mordedura de perros en niños. Arch Argent Pediatr. 2005; 103(5): 389-395.

2. Blanco M, Pérez W. Mordeduras de perro en niños. Arch Pediatr Urug. 2004; 75(2): 122-123.

3. Martínez M. Microorganismos asociados a infecciones por mordeduras de perros y gatos. Mon Electr Pato Vet. 2005; 2(1): 1-16.

4. Jofré L, Perret C, Abarca K, Solari V, Olivares R, López J. Recomendaciones para el manejo de mordeduras ocasionadas por animales. Rev Chil Infect. 2006; 23 (1): 20-34.

5. Palacio J, Leon M, Garcia - Belenguer S. Aspectos Epidemiológicos de las mordeduras caninas. Gac Sanit. 2005; 19(1): 50-58.

6. Morales C, Falcón N, Hernández H, Fernández C. Accidentes por mordedura canina, casos registrados en un hospital de niños de Lima, Perú 1995 - 2009. Rev Peru Med Exp Salud Publica. 2011; 28(4): 63942.

7. Besada A, Huaier F, Jorge F. Los niños y los perros ¿Cómo reducir los riesgos y maximizar los beneficios? Revista del Hospital de Niños Dr. Ricardo Gutiérrez. 2002; 44: 141-148.

8. Lema F. Mordedura de perro, comportamiento y agresión. Arch Argent Pediatr. 2005; 103: 5.

9. Quiles-Cosme GM, Pérez-Cardona CM, Aponte FI. Estudio descriptivo sobre ataques y mordeduras de animales en el Municipio de San Juan, Puerto Rico, 1996-1998. Health Sci J. 2000; 19: 39-47.

10. Borud LJ, Friedman DW. Dog bites in New York city. Plast Reconstr Surg. 2000; 106(5): 987-90.

11. Holmquist L, Elixhauser A. Emergency department visits and impatient says involving dog bites (Internet). Rockville, MD: Healthcare cost and Utilization Project; 2008. (Citado en Mayo del 2011) Disponible en: http://www.hcup-us.ahrq.gov/reports/statbriefs/ sb101.pdf

12. Beaver BV. Human-canine interactions: a summary of perspectives. J Am Vet Med Assoc. 1997; 210: 1148-1150.

13. Callaham M. Dog bites wounds. JAMA. 1980; 244(20): 2327-8.

14. Congreso de la Republica. Ley $N^{\circ} 27596$ Regula el régimen jurídico de canes. Lima, Perú: Diario Oficial El Peruano; 14 de Diciembre del 2001. 
15. Mondragón M, Lozano S, Hernández G, Duarte J, Álvarez P. Heridas por mordedura de perro: Tratamiento de 40 pacientes. Acta Pediatr Mex. 1997; 18(3): 120-123.

16. Trott A. Care of mammaliam bites. Pediatr Infect Dis J. 1987; 6(1): 8-10.

17. Boenning D, Fleisher G, Campos J. Dog bites in children. Epidemiology, microbiology, and penicillin prophylactic therapy. Am J Emerg Med. 1983; 1:1721.

18. Van Damme P. Surgical treatment of facial dog bite injuries in children. J Craniomaxillofac Surg. 1992; 20(8): 362-3
19. Underman A. Bite wounds inflicted by dogs and cats. Vet Clin North Am Small Anim Pract. 1987; 17(1): 195-207.

20. Brook I. Microbiology of human and animal bite wounds. Pediart Infect Dis J. 1987; 6(1): 29-32.

21. Glausiuss G, Ascione I, Sehabiague G. Mordeduras por animales en la edad pediátrica. Arch Pediatr Urug. 2000; 71(1): 24-30.

Recibido: 01/08/2013

Aceptado: 21/11/2013 